\title{
Neutrino mass matrix solutions and neutrinoless double beta decay
}

\author{
T. Hambye \\ Centre de Physique Théorique, CNRS Luminy, \\ Case 907, 13288 Marseille, France
}

\begin{abstract}
We present a determination of the neutrino mass matrix which holds for values of the neutrinoless double beta decay effective mass $m_{e e}$ larger than the neutrino mass differences. We find eight possible solutions and discuss for each one the corresponding neutrino mass eigenvalues and zero texture. A minimal structure of the perturbations to add to these zero textures to recover the full mass matrix is also determined. Implications for neutrino hot dark matter are discussed for each solution.
\end{abstract}


1) Introduction: The neutrino mass matrix in the three neutrino framework has been severely constrained over the last few years by atmospheric [1] and solar [2] neutrino experiments, and by recent positive indications from laboratory experiments [3, [4]. In the basis in which the charged lepton mass matrix is diagonal, the neutrino mass matrix contains all the information about the mixing schemes in the leptonic sector. The mixing angle required for the atmospheric neutrinos appears to be maximal or very close to maximal. This is one of the main constraints on the neutrino mass matrix, when combined with the mass squared difference required for atmospheric neutrinos $\delta m_{\text {atm }}^{2}$, which is in the range $(1.5-4.8) \cdot 10^{-3}[5]$. There are a few solutions to the solar neutrinos, which give mass squared differences $\delta m_{\text {sol }}^{2}$ and mixing angle $\theta$ with $\nu_{e}$ which can lie in various ranges (see e.g. Ref. [6]). The reactor experiment $\mathrm{CHOOZ}$ gives in addition a strong upper limit on the $U_{e 3}$ element of the neutrino mixing matrix [7]. Besides these five constraints on the neutrino mass matrix, a sixth one would be given by the measurement of the effective mass $m_{e e}$ in neutrinoless double beta decay. This parameter would provide a scale to the neutrino mass matrix. Very recently a positive indication of neutrinoless double has been reported with $m_{e e}$ in the following range [8]:

$$
\left.m_{e e}=(0.05-0.86) \mathrm{eV} \text { (at } 95 \% \text { c.l. }\right)
$$

and with a best fit for $m_{e e}=0.39 \mathrm{eV}$. In the following we study what could be the consequences for the neutrino mass matrix of a value of $m_{e e}$ within the range of Eq. (1). By considering this range we study more generally what would be the effect on the mass matrix of an effective mass $m_{e e}$ determined with a value greater than the neutrino mass differences and smaller than the dark matter constraints (see below). This range covers also the values of $m_{e e}$ which could be further tested experimentally in a relatively near future [9]. Since the scale $m_{e e}$ is then higher than the mass squared differences required by the neutrino oscillation results, this implies a degenerate or partially degenerate solution [10]. The hierarchical solution is not allowed by a value of $m_{e e}$ within the range of Eq. (11). An inverse hierarchy solution is still possible but only for $m_{e e}$ close to the lower edge of this range.

Considering the fact that the atmospheric mixing is close to maximal, several texture mass matrices have been constructed [11], including in particular the ones where the mass squared difference $\delta m_{a t m}^{2}$ is considered to be the dominant entry in the mass matrix and where the solar neutrino solutions could be added as perturbations. These textures are very useful to construct models, where the zeroes could have their origin in some symmetry of the model, so that the perturbations come from the symmetry breaking. For a value of $m_{e e}$ within the range of Eq. (11), $\delta m_{\text {atm }}^{2}$ would likely becomes a perturbation in the neutrino 
mass matrix with respect to the scale $m_{e e}$. This would be an important information for the determination of the texture of the mass matrix. Moreover, given a value of $m_{e e}$ in the range of Eq. (II) the neutrino contribution to the hot dark matter would be significant. With these assumptions we now attempt to look into this problem. Instead of starting the analysis from the possible textures, we start with the experimental inputs and then try to determine the neutrino mass matrices and in the process get the possible textures.

In the following we shall work in the basis in which the charged lepton mass matrix is diagonal. The physical states $\mid \nu_{\alpha}>,(\alpha=e, \mu, \tau)$, which enter in the charged current, are related to the mass eigenstates $\mid \nu_{i}>$, (with masses $m_{i}, i=1,2,3$ ), by the mixing matrix

$$
\left|\nu_{\alpha}>=U_{\alpha i}\right| \nu_{i}>
$$

and the effective mass entering in the neutrinoless double beta decay is

$$
m_{e e}=\left(M_{\nu}\right)_{e e}=\left(M_{\nu}\right)_{11}=\sum_{i} U_{e i}^{2} m_{i}
$$

$\left(M_{\nu}\right)_{11}$ is the $(11)$ element of the neutrino mass matrix in the flavour basis.

In this article we do not consider the imaginary part of the mass matrix assuming that there is no $\mathrm{CP}$ violation, or very small $\mathrm{CP}$ violation. In this limit, we can parametrize the mass matrix in the flavor basis as a function of the three real mass eigenvalues and the three angles coming from the mixing matrix $U_{\alpha i}$. For simplicity and also as a first step of a more involved analysis we consider in addition only the case with exact maximal mixing between the $\nu_{\mu}$ and $\nu_{\tau}$ (motivated by the best fit value from SuperKamiokande) and we consider $U_{e 3}=0$ (motivated by the result of the CHOOZ experiment). In this framework we determine what are the effects on these six mass matrix parameters of the five experimental constraints mentioned above taking a given value of $m_{e e}$. We find that there are eight different solutions for the mass matrix, each one being determined by these six constraints. For each solution we display the corresponding zero texture and show how the perturbations arise around these zero textures to give the full mass matrix. We also discuss the consequences for hot dark matter for the different solutions.

2) The neutrino mass matrix solutions: In a general way the diagonal neutrino mass matrix can be written as:

$$
M_{\nu}^{\text {diag }}=\left(\begin{array}{ccc}
m_{1} & 0 & 0 \\
0 & m_{2} e^{i \phi_{2}} & 0 \\
0 & 0 & m_{3} e^{i \phi_{3}}
\end{array}\right)
$$


here $\phi_{2,3}$ are the Majorana phases, which introduces $\mathrm{CP}$ violation in lepton number violating processes. The mixing matrix $U_{\alpha i}$ may be parametrized as

$$
U=\left(\begin{array}{ccc}
c_{1} c_{3} & -s_{1} c_{3} & -s_{3} e^{-i \phi} \\
s_{1} c_{2}-c_{1} s_{2} s_{3} e^{i \phi} & c_{1} c_{2}+s_{1} s_{2} s_{3} e^{i \phi} & -c_{3} s_{2} \\
s_{1} s_{2}+c_{1} c_{2} s_{3} e^{i \phi} & c_{1} s_{2}-s_{2} c_{2} s_{3} e^{i \phi} & c_{2} c_{3}
\end{array}\right)
$$

where, $s_{i}=\sin \theta_{i}$ and $c_{i}=\cos \theta_{i}$. This depends on 3 mixing angles $\theta_{i}$ and one CP-violating phase $\phi$. Thus there are 9 parameters in the general mass matrix in the flavor basis [12].

The atmospheric neutrinos require an almost maximal mixing between the flavour states $\nu_{\mu}$ and $\nu_{\tau}$. A very little mixing with the $\nu_{e}$ is not ruled out, but it is constrained by the $\mathrm{CHOOZ}$ result. Considering this constraint and the fact that the central value of the mixing angle for atmospheric neutrinos is maximal, as required by the SuperKamiokande result and supported by the K2K preliminary data, we assume only maximal mixing $s_{2}=1 / \sqrt{2}$ between the flavour states $\nu_{\mu}$ and $\nu_{\tau}$. Although the CHOOZ constraint allows a small $U_{e 3}$, for construction of a simple form of a mass matrix we assume it to be vanishing, $s_{3}=0$. In this limit of $U_{e 3}=0$ the CP-violating effect from the phase $\phi$ in $U$ vanishes and we can neglect its effect. We can then write down the mixing matrix in terms of the mixing angle $\theta=\theta_{1}$ required for the solar neutrinos solutions as

$$
U=\left(\begin{array}{ccc}
c & -s & 0 \\
s / \sqrt{2} & c / \sqrt{2} & -1 / \sqrt{2} \\
s / \sqrt{2} & c / \sqrt{2} & 1 / \sqrt{2}
\end{array}\right)
$$

with $c=\cos \theta, s=\sin \theta$.

This leads to the following mass matrix in the $\nu_{e}, \nu_{\mu}, \nu_{\tau}$ flavor basis:

$$
M_{\nu}=\left(\begin{array}{ccc}
c^{2} m_{1}+s^{2} m_{2} & \frac{c s}{\sqrt{2}}\left(m_{1}-m_{2}\right) & \frac{c s}{\sqrt{2}}\left(m_{1}-m_{2}\right) \\
\frac{c s}{\sqrt{2}}\left(m_{1}-m_{2}\right) & \frac{s^{2} m_{1}+c^{2} m_{2}+m_{3}}{2} & \frac{s^{2} m_{1}+c^{2} m_{2}-m_{3}}{2} \\
\frac{c s}{\sqrt{2}}\left(m_{1}-m_{2}\right) & \frac{s^{2} m_{1}+c^{2} m_{2}-m_{3}}{2} & \frac{s^{2} m_{1}+c^{2} m_{2}+m_{3}}{2}
\end{array}\right),
$$

In the following we disregard the effect of the Majorana phases $\phi_{2,3}$ of the diagonal mass matrix. These CP violation does not enter in any neutrino oscillation experiments. We will consider only real (positive or negative) mass eigenvalues. By considering both positive and negative mass eigenvalues we consider the extreme cases, and the case with non-zero phases are intermediate cases between these two extremes. The results with phases can be obtained as a simple generalization of what we present here. Under these assumptions, to cover all physical distinct possibilities without loss of generality, it is sufficient to take $m_{e e}$ positive and $\left|m_{2}\right|>\left|m_{1}\right|$ by definition and to consider the range $0^{\circ}<\theta<90^{\circ}$. Then 
from the value of $\delta m_{a t m}^{2}, \delta m_{\text {sol }}^{2}, \theta$, and $m_{e e}$ we can determine the mass matrix. There are 8 solutions. To find them we have first to solve the equations:

$$
\begin{gathered}
m_{2}^{2}-m_{1}^{2}=\delta m_{\text {sol }}^{2} \\
m_{e e}=m_{1} c^{2}+m_{2} s^{2}
\end{gathered}
$$

This gives two sets of solutions:

$$
\begin{aligned}
& m_{1}^{A}=m_{e e}-\frac{s^{2}}{2} \frac{\delta m_{\text {sol }}^{2}}{m_{e e}} \\
& m_{2}^{A}=m_{e e}+\frac{c^{2}}{2} \frac{\delta m_{\text {sol }}^{2}}{m_{e e}}
\end{aligned}
$$

and

$$
\begin{aligned}
& m_{1}^{B}=\frac{m_{e e}}{\cos 2 \theta}+\frac{s^{2}}{2} \frac{\delta m_{s o l}^{2}}{m_{e e}} \\
& m_{2}^{B}=-\frac{m_{e e}}{\cos 2 \theta}-\frac{c^{2}}{2} \frac{\delta m_{s o l}^{2}}{m_{e e}}
\end{aligned}
$$

where one can safely keep only the first order in $\delta m_{s o l}^{2} / m_{e e}^{2}$ as we did here. Then we can determine the mass $m_{3}$ from the equation:

$$
m_{3}^{2}=\frac{m_{1}^{2}+m_{2}^{2}}{2} \pm \delta m_{\text {atm }}^{2}
$$

From this we find four solutions with the set $m_{1,2}^{A}$ :

$$
\begin{aligned}
& m_{3}^{A_{1}, A_{2}}=\sqrt{m_{e e}^{2} \pm \delta m_{a t m}^{2}} \\
& m_{3}^{A_{3}, A_{4}}=-\sqrt{m_{e e}^{2} \pm \delta m_{a t m}^{2}}
\end{aligned}
$$

and four solutions with the set $m_{1,2}^{B}$ :

$$
\begin{aligned}
& m_{3}^{B_{1}, B_{2}}=-\sqrt{\frac{m_{e e}^{2}}{\cos ^{2} 2 \theta} \pm \Delta m_{a t m}^{2}} \\
& m_{3}^{B_{3}, B_{4}}=+\sqrt{\frac{m_{e e}^{2}}{\cos ^{2} 2 \theta} \pm \Delta m_{a t m}^{2}}
\end{aligned}
$$

Here in $m_{3}$ we kept only the correction in $\delta m_{a t m}^{2} / m_{e e}^{2}$ and neglected the tiny correction from $\delta m_{s o l}^{2}$.

3) Neutrino mass numerical values: Putting Eqs. (10)-(18) in Eq. (7), we can directly build the eight possible matrices and substitute the values of $m_{e e}, \delta m_{a t m}^{2}, \delta m_{\text {sol }}^{2}$ and $\theta$ to 


\begin{tabular}{|c|c||c|c|}
\hline \multirow{4}{*}{$A_{1}$} & $m_{1}=0.390$ & & $m_{1}=0.848$ \\
& $m_{2}=0.390$ & & $m_{2}=-0.848$ \\
& $m_{3}=0.394$ & $B_{1}$ & $m_{3}=-0.850$ \\
& $0.050 \leq m_{1} \leq 0.86$ \\
& $0.050 \leq m_{2} \leq 0.86$ & & $0.0750 \leq m_{1} \leq 7.74$ \\
& $0.0632 \leq m_{3} \leq 0.8628$ & & $-7.74 \leq m_{2} \leq-0.0750$ \\
& $m_{1}=0.390$ & & \\
\hline \hline \multirow{4}{*}{$A_{2}$} & $m_{2}=0.390$ & & $m_{1}=0.848$ \\
& $m_{3}=0.386$ & $B_{2}$ & $m_{3}=-0.848$ \\
& $0.049 \leq m_{1} \leq 0.860$ & & $0.0750 \leq m_{3} \leq-0.0845$ \\
& $0.050 \leq m_{2} \leq 0.860$ & & $-7.74 \leq m_{2} \leq-0.075$ \\
& $0 \leq m_{3} \leq 0.859$ & & $-7.74 \leq m_{3} \leq-0.029$ \\
\hline
\end{tabular}

Table 1: Central values and allowed ranges of the mass eigenvalues $m_{1,2,3}$ (in eV). For the solutions $A_{3}, A_{4}, B_{3}, B_{4}$, the mass eigenvalues are the same as that of the solutions $A_{1}, A_{2}$, $A_{3}, A_{4}$, respectively, except for the sign of $m_{3}$ which has to be reversed. We put several digits just to show the effect of $\delta m_{a t m}^{2}$ which is different for the various solutions.

obtain the possible matrices. To illustrate the differences between the various solutions, we give in Table 1 the values of the masses $m_{1,2,3}$ obtained in the eight cases, taking, as a representative value of $m_{e e}$, the central value from [8], $m_{e e}=0.39 \mathrm{eV}$, and taking for the other parameters the following central values in the case of the LMA solution: $\delta m_{\text {atm }}^{2}=2.9 \cdot 10^{-3} \mathrm{eV}^{2}$ [0] $\delta m_{\text {sol }}^{2}=3.7 \cdot 10^{-5} \mathrm{eV}^{2}$ [6], $\tan ^{2} \theta=0.37$ [6]. The uncertainties on these masses due to the experimental uncertainties on these four parameters are also given in Table 1 . To get them we took the following ranges: $\delta m_{\text {atm }}^{2}=(1.5-4.8) \cdot 10^{-3} \mathrm{eV}^{2}$ [5], $\delta m_{\text {sol }}^{2}=(0.2-3) \cdot 10^{-4} \mathrm{eV}^{2}$ [6], $\tan ^{2} \theta=0.2-0.8$ [6], $m_{e e}=(0.05-0.86) \mathrm{eV}$ [8]. The uncertainties on $U_{e 3}$ and the atmospheric angle also induce an error, which, however, is moderate and which we didn't consider. We will discuss these central values and ranges in sections 5 and 6 .

For the SMA solution, although this solution is now almost ruled out [6], the same calculation can be done and for completeness we give in Table 2 the values of $m_{1,2,3}$ we obtain considering the following central values from Ref. $[6]$ : $\delta m_{\text {sol }}^{2}=5.2 \cdot 10^{-6} \mathrm{eV}^{2}$ and $\tan ^{2} \theta=1.8 \cdot 10^{-3}$. We don't give here any range because the central values for the SMA 


\begin{tabular}{|c|c||c|c|}
\hline \multirow{3}{*}{$A_{1}$} & $m_{1}=0.390$ & & $m_{1}=0.391$ \\
& $m_{2}=0.390$ & $B_{1}$ & $m_{2}=-0.391$ \\
& $m_{3}=0.394$ & & $m_{3}=-0.395$ \\
\hline \hline & $m_{1}=0.390$ & & $m_{1}=0.391$ \\
$A_{2}$ & $m_{2}=0.390$ & $B_{2}$ & $m_{2}=-0.391$ \\
& $m_{3}=0.386$ & & $m_{3}=-0.388$ \\
\hline
\end{tabular}

Table 2: Central values of the mass eigenvalues $m_{1,2,3}$ (in eV) for the SMA solution. For the solutions $A_{3}, A_{4}, B_{3}, B_{4}$, the mass eigenvalues are the same than for the solutions $A_{1}$, $A_{2}, A_{3}, A_{4}$ respectively except for the sign of $m_{3}$ which has to be reversed.

solution have been obtained in Ref. [6] beyond the 3- $\sigma$ level. The results for the LOW and VAC solar neutrino solutions can be obtained in the same way but we don't present it because they are in general difficult to accommodate with a value of $m_{e e}$ larger than the neutrino mass differences [10]. See however counter-examples in Ref. [13].

4) Zero textures and perturbations: To understand better the structure of these matrices and also for the purpose of model building it is interesting to write these matrices in the form of textures zero plus the perturbations. This will also allow us to compare our results with the textures predicted from different considerations [11. In the following we shall consider the degenerate solution assuming that $m_{e e}$ is larger than the neutrino mass differences. For the inverse hierarchy solution we will just give a few comments in sections 5 and 6.

In the case where the mass eigenvalues are almost degenerate with the mass squared differences to be small compared to the eigenvalues, i.e. $\left|m_{1,2,3}\right| \gg \delta m_{\text {atm }}^{2} \gg \delta m_{\text {sol }}^{2}$, the parameters $\delta m_{\text {atm }}^{2}$ and $\delta m_{\text {sol }}^{2}$ can be considered as perturbations with respect to $m_{e e} \sim$ $\left|m_{1,2,3}\right|$. In this case we get the following zero textures and perturbations for the different solutions:

$$
\begin{aligned}
M_{A_{1}, A_{2}} & =m_{e e}\left(\begin{array}{lll}
1 & 0 & 0 \\
0 & 1 & 0 \\
0 & 0 & 1
\end{array}\right) \pm m_{e e} \frac{\varepsilon_{a t m}}{4}\left(\begin{array}{ccc}
0 & 0 & 0 \\
0 & 1 & -1 \\
0 & -1 & 1
\end{array}\right) \\
& +m_{e e} \frac{\varepsilon_{\text {sol }}}{8}\left(\begin{array}{ccc}
0 & -\sqrt{2} \sin 2 \theta & -\sqrt{2} \sin 2 \theta \\
-\sqrt{2} \sin 2 \theta & 2 \cos 2 \theta & 2 \cos 2 \theta \\
-\sqrt{2} \sin 2 \theta & 2 \cos 2 \theta & 2 \cos 2 \theta
\end{array}\right)
\end{aligned}
$$




$$
\begin{aligned}
& M_{A_{3}, A_{4}}=m_{e e}\left(\begin{array}{ccc}
1 & 0 & 0 \\
0 & 0 & 1 \\
0 & 1 & 0
\end{array}\right) \pm m_{e e} \frac{\varepsilon_{a t m}}{4}\left(\begin{array}{ccc}
0 & 0 & 0 \\
0 & -1 & +1 \\
0 & +1 & -1
\end{array}\right) \\
& +m_{e e} \frac{\varepsilon_{\text {sol }}}{8}\left(\begin{array}{ccc}
0 & -\sqrt{2} \sin 2 \theta & -\sqrt{2} \sin 2 \theta \\
-\sqrt{2} \sin 2 \theta & 2 \cos 2 \theta & 2 \cos 2 \theta \\
-\sqrt{2} \sin 2 \theta & 2 \cos 2 \theta & 2 \cos 2 \theta
\end{array}\right) \text {, } \\
& M_{B_{1}, B_{2}}=\frac{m_{e e}}{\cos 2 \theta}\left(\begin{array}{ccc}
\cos 2 \theta & \frac{\sin 2 \theta}{\sqrt{2}} & \frac{\sin 2 \theta}{\sqrt{2}} \\
\frac{\sin 2 \theta}{\sqrt{2}} & -\frac{1}{2}-\frac{\cos 2 \theta}{2} & \frac{1}{2}-\frac{\cos 2 \theta}{2} \\
\frac{\sin 2 \theta}{\sqrt{2}} & \frac{1}{2}-\frac{\cos 2 \theta}{2} & -\frac{1}{2}-\frac{\cos 2 \theta}{2}
\end{array}\right) \\
& \pm m_{e e} \cos 2 \theta \frac{\varepsilon_{a t m}}{4}\left(\begin{array}{ccc}
0 & 0 & 0 \\
0 & -1 & +1 \\
0 & +1 & -1
\end{array}\right) \\
& +m_{e e} \frac{\varepsilon_{\text {sol }}}{8}\left(\begin{array}{ccc}
0 & \sqrt{2} \sin 2 \theta & \sqrt{2} \sin 2 \theta \\
\sqrt{2} \sin 2 \theta & -2 \cos 2 \theta & -2 \cos 2 \theta \\
\sqrt{2} \sin 2 \theta & -2 \cos 2 \theta & -2 \cos 2 \theta
\end{array}\right) \\
& M_{B_{3}, B_{4}}=\frac{m_{e e}}{\cos 2 \theta}\left(\begin{array}{ccc}
\cos 2 \theta & \frac{\sin 2 \theta}{\sqrt{2}} & \frac{\sin 2 \theta}{\sqrt{2}} \\
\frac{\sin 2 \theta}{\sqrt{2}} & \frac{1}{2}-\frac{\cos 2 \theta}{2} & -\frac{1}{2}-\frac{\cos 2 \theta}{2} \\
\frac{\sin 2 \theta}{\sqrt{2}} & -\frac{1}{2}-\frac{\cos 2 \theta}{2} & \frac{1}{2}-\frac{\cos 2 \theta}{2}
\end{array}\right) \\
& \pm m_{e e} \cos 2 \theta \frac{\varepsilon_{a t m}}{4}\left(\begin{array}{ccc}
0 & 0 & 0 \\
0 & 1 & -1 \\
0 & -1 & 1
\end{array}\right) \\
& +m_{e e} \frac{\varepsilon_{\text {sol }}}{8}\left(\begin{array}{ccc}
0 & \sqrt{2} \sin 2 \theta & \sqrt{2} \sin 2 \theta \\
\sqrt{2} \sin 2 \theta & -2 \cos 2 \theta & -2 \cos 2 \theta \\
\sqrt{2} \sin 2 \theta & -2 \cos 2 \theta & -2 \cos 2 \theta
\end{array}\right) \text {, }
\end{aligned}
$$

Here $\varepsilon_{\text {sol }} \equiv \delta m_{\text {sol }}^{2} / m_{e e}^{2}$ and $\varepsilon_{a t m} \equiv \delta m_{a t m}^{2} / m_{e e}^{2}$. The corresponding mass eigenvalues are given in Table 3 . Note that the various solutions differ from each other by their zero texture and by prefactors in the perturbations. Except for these different prefactors the various perturbations have the same matrix structure for all the mass matrix solutions.

5) The $A_{1,2,3,4}$ solutions: First let us discuss the solutions $A_{1}$ to $A_{4}$ : 


\begin{tabular}{|c|c|c|c|c|}
\hline & \multicolumn{3}{|c|}{$M_{(0)}^{\nu}$} & $m_{1,2,3}$ \\
\hline$A_{1,2}$ & & $e\left(\begin{array}{ll}1 & 0 \\
0 & 1 \\
0 & 0\end{array}\right.$ & $\left.\begin{array}{l}0 \\
0 \\
1\end{array}\right)$ & $\begin{array}{c}m_{1}=m_{e e}\left(1-s^{2} \frac{\varepsilon_{s o l}}{2}\right) \\
m_{2}=m_{e e}\left(1+c^{2} \frac{\varepsilon_{s o l}}{2}\right) \\
m_{3}=m_{e e}\left(1 \pm \frac{\varepsilon_{a t m}}{2}\right)\end{array}$ \\
\hline$A_{3,4}$ & & $e\left(\begin{array}{ll}1 & 0 \\
0 & 0 \\
0 & 1\end{array}\right.$ & $\left.\begin{array}{l}0 \\
1 \\
0\end{array}\right)$ & $\begin{array}{l}m_{1}=m_{e e}\left(1-s^{2} \frac{\varepsilon_{s o l}}{2}\right) \\
m_{2}=m_{e e}\left(1+c^{2} \frac{\varepsilon_{s o l}}{2}\right) \\
m_{3}=-m_{e e}\left(1 \pm \frac{\varepsilon_{a t m}}{2}\right)\end{array}$ \\
\hline$B_{1,2}$ & $\tilde{m}\left(\begin{array}{c}\cos 2 \theta \\
\frac{\sin 2 \theta}{\sqrt{2}} \\
\frac{\sin 2 \theta}{\sqrt{2}}\end{array}\right.$ & $\begin{array}{c}\frac{\sin 2 \theta}{\sqrt{2}} \\
-\frac{1}{2}-\frac{\cos 2 \theta}{2} \\
\frac{1}{2}-\frac{\cos 2 \theta}{2}\end{array}$ & $\begin{array}{c}\frac{\sin 2 \theta}{\sqrt{2}} \\
\frac{1}{2}-\frac{\cos 2 \theta}{2} \\
-\frac{1}{2}-\frac{\cos 2 \theta}{2}\end{array}$ & $\begin{array}{c}m_{1}=\tilde{m}\left(1+s^{2} \cos 2 \theta \frac{\varepsilon_{s o l}}{2}\right) \\
m_{2}=-\tilde{m}\left(1+c^{2} \cos 2 \theta \frac{\varepsilon_{s o l}}{2}\right) \\
m_{3}=-\tilde{m}\left(1 \pm \cos ^{2} 2 \theta \frac{\varepsilon_{a t m}}{2}\right)\end{array}$ \\
\hline$B_{3,4}$ & $\tilde{m}\left(\begin{array}{c}\cos 2 \theta \\
\frac{\sin 2 \theta}{\sqrt{2}} \\
\frac{\sin 2 \theta}{\sqrt{2}}\end{array}\right.$ & $\begin{array}{c}\frac{\sin 2 \theta}{\sqrt{2}} \\
\frac{1}{2}-\frac{\cos 2 \theta}{2} \\
-\frac{1}{2}-\frac{\cos 2 \theta}{2}\end{array}$ & $\begin{array}{c}\frac{\sin 2 \theta}{\sqrt{2}} \\
-\frac{1}{2}-\frac{\cos 2 \theta}{2} \\
\frac{1}{2}-\frac{\cos 2 \theta}{2}\end{array}$ & $\begin{array}{c}m_{1}=\tilde{m}\left(1+s^{2} \cos 2 \theta \frac{\varepsilon_{s o l}}{2}\right) \\
m_{2}=-\tilde{m}\left(1+c^{2} \cos 2 \theta \frac{\varepsilon_{s o l}}{2}\right) \\
m_{3}=\tilde{m}\left(1 \pm \cos ^{2} 2 \theta \frac{\varepsilon_{a t m}}{2}\right)\end{array}$ \\
\hline
\end{tabular}

Table 3: Zero textures and neutrino mass eigenvalues at first order in $\varepsilon_{\text {atm }}$ and $\varepsilon_{\text {sol }}$ corresponding to the solutions $A_{1,2,3,4}$ and to $B_{1,2,3,4}$.

- Mass eigenvalues: For these solutions the absolute values of the masses of the 3 eigenstates coincide with $m_{e e}$ modulo small variations (proportional to $\delta m_{a t m}^{2}$ and $\delta m_{\text {sol }}^{2}$ ). This explains why in Table 1 the upper limit of the neutrino mass ranges reproduce to a good approximation the upper limit on $m_{e e}$. For low values of $m_{e e}$ (around $0.05 \mathrm{eV}$ in Eq. (11)), the neutrino masses $m_{1,2}$ are still given to a good approximation by $m_{e e}$ but the $\left|m_{3}\right|$ value can differ substantially from the one of $m_{e e}$. This is due to the fact that in this case $\delta m_{a t m}^{2}$ is not small anymore with respect to $m_{e e}$ and $\left|m_{3}\right|$ could be as low as $0 \mathrm{eV}$ for the solutions $A_{2,4}$ (where $\delta m_{\text {atm }}^{2}$ and $m_{e e}$ come with opposite signs in $m_{3}$ ). This later case corresponds to the inverse hierarchy scenario.

- Dark matter: For a value of $m_{e e}$ such as the central value $m_{e e}=0.39 \mathrm{eV}$ [8 or above, the solutions $A_{1,2,3,4}$ could be relevant for dark matter. $\left|m_{1}\right|=\left|m_{2}\right|=\left|m_{3}\right|=$ $m_{e e}=0.39 \mathrm{eV}$ (or the upper value $0.86 \mathrm{eV}$ in Eq. (1)) corresponds, in units of the critical density, to a value of $\Omega_{\nu} h^{2} \sim \sum\left|m_{i}\right| /(91.5 \mathrm{eV})$ around $\sim 1.5 \%(\sim 3 \%)$ with 
$h$ the dimensionless Hubble constant. Note that the value $\Omega_{\nu} h^{2} \sim 3 \%$ turns out to be about the upper bound allowed by fits from observed galaxy clustering and the Lyman $\alpha$ forest (see e.g. [15]-18] and the discussion in [10]).

- Textures and perturbations in the degenerate case: For the cases $A_{1,2}$ and $A_{3,4}$ we recognize the textures $\mathrm{C} 0$ and $\mathrm{C} 3$ of Altarelli-Feruglio, respectively [11](naturally leading to both simple maximal mixing or double maximal mixing). From Eqs. (7)(18) one can observe also how the perturbations arise with respect to these zero textures to give the full mass matrix. Note that the structure of the perturbations due to $\delta m_{a t m}^{2}$ is quite simple, which is interesting for model building. Note also that in case $A_{1,2}$ it is this perturbation which is responsible for the maximal atmospheric mixing. In case $A_{3,4}$ the maximal mixing is already present for $\delta m_{\text {atm }}^{2}=0$. The structure of the perturbation from $\delta m_{\text {sol }}^{2}$ is more complicated but remains rather simple especially if $\theta$ takes a specific value (i.e. for example for $\theta=0$ or $\theta=\pi / 4$ ). In all cases $A_{1}$ to $A_{4}$ the solar mixing is due to the perturbation proportional to $\delta m_{\text {sol }}^{2}$. The solar angle even if maximal is a perturbation because all the terms depending on it are multiplied by $\delta m_{s o l}^{2}$. There is no solar mixing if $\delta m_{\text {sol }}^{2}$ vanishes.

- The inverse hierarchy case: If $m_{e e}$ turns out to be not much larger than the lower edge of the experimental range in Eq. (1), i.e. $m_{e e}=0.05 \mathrm{eV}$, we could have an inverse hierarchy where $\left|m_{3}\right|<<\left|m_{1,2}\right|$ which means, since $m_{e e}^{2}>>\delta m_{s o l}^{2}$, that $\left|m_{3}\right|<<\left|m_{e e}\right|$. Such a situation requires a cancellation in $m_{3}$, Eqs. (15) and (16), of the $m_{e e}$ term with the term proportional to $\delta m_{a t m}^{2}$. This is possible only with solutions $A_{2,4}$ where $\delta m_{\text {atm }}^{2}$ comes with an opposite sign with respect to the term in $m_{e e}^{2}$ in $m_{3}$. For these solutions this requires $m_{e e}^{2} \sim \delta m_{a t m}^{2}$. For the central values $\delta m_{\text {atm }}^{2}=2.9 \cdot 10^{-3} \mathrm{eV}^{2}$ [5] and $\tan ^{2} \theta=0.37$ [6] this requires $m_{e e} \sim 0.054 \mathrm{eV}$. Varying $\delta m_{\text {atm }}^{2}$ within the range $(1.5-4.8) \cdot 10^{-3} \mathrm{eV}^{2}$ this requires:

$$
4 \cdot 10^{-2} \mathrm{eV}<m_{e e}<7 \cdot 10^{-2} \mathrm{eV} \text { (for solutions } A_{2,4} \text { ) }
$$

This range is independent of the solar neutrino solution. Note also that we could develop in this case the mass matrix considering $m_{3}$ and $\sqrt{\delta m_{\text {sol }}^{2}}$ as perturbations with respect to $m_{e e}$ and $\sqrt{\delta m_{a t m}^{2}}$. In this case the zero texture we obtain is

$$
\left(\begin{array}{ccc}
1 & 0 & 0 \\
0 & 1 / 2 & 1 / 2 \\
0 & 1 / 2 & 1 / 2
\end{array}\right)
$$

that is to say the texture B2 of Ref. [11] which can lead to both single and double maximal mixing. 
6) The $B_{1,2,3,4}$ solutions: Solutions $B_{1}$ to $B_{4}$ are very different from $A_{1}$ to $A_{4}$ and more complicated because $m_{1}$ and $m_{2}$ here have opposite sign (c.f. Table 3 ):

- Neutrino mass eigenvalues: Here in the limit $\delta m_{a t m}^{2}=\delta m_{\text {sol }}^{2}=0$ the absolute values of the neutrino masses are not $m_{e e}$ (as with solutions $A_{1}$ to $A_{4}$ ) but turn out to be in fact $\tilde{m} \equiv m_{e e} / \cos 2 \theta$. This explains the large value of the neutrino masses obtained in Table 1 with these solutions. Taking the central value $\tan ^{2} \theta \sim 0.39$ [6] for the LMA solution we then get for the neutrino masses $\tilde{m} \sim 2.2 m_{e e}$. For a value of $m_{e e}=0.39 \mathrm{eV}$ this gives $\tilde{m} \sim 0.85 \mathrm{eV}$. Taking the upper limit $\tan ^{2} \theta \sim 0.8$ [6] we get $\tilde{m} \sim 9 m_{e e}$ which, for $m_{e e}=0.86 \mathrm{eV}$ in Eq. (I), could result in a neutrino mass as large as $8 \mathrm{eV}$. However, a neutrino mass greater than $2.8 \mathrm{eV}$ is excluded by the tritium beta decay experiment 19].

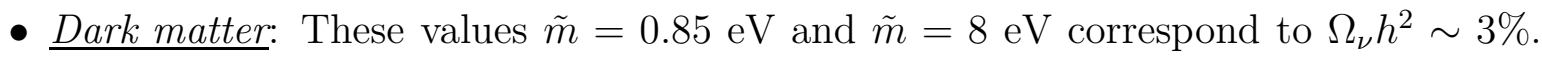
and to $\Omega_{\nu} h^{2} \sim 25 \%$ respectively. From Table 1 we then get the following range for solutions $B_{1,2,3,4}$ :

$$
0.001<\Omega_{\nu} h^{2}<25 \%
$$

Note that, as already mentioned above, a value of $\Omega_{\nu} h^{2}$ larger than $\sim 3 \%$ which corresponds to $\sum m_{i} \sim 3 \mathrm{eV}$ is disfavoured by the results of galaxy clustering and Lyman $\alpha$ forest experiments [15]- 18]. In case of the solutions $B_{1,2,3,4}$, to have $\Omega_{\nu} h^{2}$ smaller than $\sim 3 \%$ we need that the following constraint is fulfilled:

$$
\tilde{m}=\frac{m_{e e}}{\cos 2 \theta}<1 \mathrm{eV}
$$

For example this condition for $m_{e e}=0.86 \mathrm{eV}$ in Eq. (11) requires $\tan ^{2} \theta<0.08$, which can be compared with the lower limit of the LMA solution which is $\tan ^{2} \theta \sim 0.2$ [6]. This means that a value of $m_{e e}$ as large as $0.86 \mathrm{eV}$ with the solutions $B_{1,2,3,4}$ is disfavoured by dark matter fits. To obtain $\tilde{m}<1 \mathrm{eV}$ with for example $\tan ^{2} 2 \theta=$ $0.2,0.39,0.8$ we need $m_{e e}<0.7 \mathrm{eV}, 0.4 \mathrm{eV}, 0.1 \mathrm{eV}$ respectively. Would we allow $\Omega_{\nu} h^{2}$ to be as large as $10 \%$, these bounds would be multiplied obviously by a factor 3 . Note that for a $\Lambda$ CHDM model with both hot and cold dark matter as well as a cosmological constant a value of $\tilde{m}<0.5 \mathrm{eV}$ is favoured [20]. This already favours a lower value for the neutrinoless double beta decay in this scheme. Furthermore, the CMB studies with MAP and PLANCK and the Sloan Digital Sky Survey experiment (see e.g. [17]-21]) will be able to probe the mass range $\tilde{m} \sim 0.25-0.5 \mathrm{eV}$. If the contribution to the hot dark matter from neutrinos is constrained to be less than $\tilde{m}<0.25 \mathrm{eV}$, then this will mean that this class of solutions will be further restricted 
and a lower value of the neutrinoless double beta decay will be favoured. Note also that the fact that the LMA solution in the degenerate case could be relevant for dark matter is well known [14]. Here we emphasize that, taking all the experimental constraints on $U_{e 3}, \delta m_{a t m}^{2}, \delta m_{\text {solar }}^{2}$ and taking $m_{e e}$ within the range of Eq. (1), all the solutions for the mass matrix, and especially the $B_{1,2,3,4}$ solutions lead naturally to such a rather important contribution to dark matter. Inversely the dark matter constraint prefers a lower value of the $m_{e e}$ or a higher $\cos 2 \theta$ for the solutions $B_{1,2,3,4}$. Note also that for the SMA solution with the central values $\delta m_{\text {sol }}^{2}=5.2 \cdot 10^{-5} \mathrm{eV}^{2}$ and $\tan ^{2} \theta=1.8 \cdot 10^{-3}$ we obtain $\tilde{m}=m_{e e}$ to a good approximation which means for $m_{e e}=0.39 \mathrm{eV}$ that $\Omega_{\nu} h^{2}=1.5 \%$.

- Textures and perturbations in the degenerate case: In contrast with solutions $A_{1,2,3,4}$ the dependence in $\theta$ is already non-vanishing even without any perturbation proportional to $\delta m_{\text {sol }}^{2}$ and $\delta m_{\text {atm }}^{2}$. So even in this limit there is in fact a "continuum" of zero textures depending on the value of $\theta$, see Eqs. (21) and (22). This allows for a continuum of basis where the zero texture is diagonal, i.e. of basis which can be considered as natural in the symmetry limit. Eventually for some particular values of the angle $\theta$ the corresponding diagonal mass matrix will display interesting properties. These considerations are beyond the scope of this article.

The structure of the perturbations appear to be also more complicated than for $A_{1,2,3,4}$. In particular, now there is a dependence of the perturbation from $\delta m_{a t m}^{2}$ in $\theta$, which would lead to the interpretation that this perturbation cannot be independent of the other perturbations, unlike in the solutions $A_{1,2,3,4}$ where they are independent. In the continuum of possible zero textures for the $B_{1}$ to $B_{4}$ solutions, a natural choice is to take the textures with $\theta=0$ or $\theta=\pi / 4$, and make a perturbation around these cases (for the SMA and the LMA respectively). In this case the deviation of $\theta$ from these values can be considered as a perturbation. For $\theta=0$ we obtain:

$$
\begin{aligned}
M_{B_{1}, B_{2}} & =m_{e e}\left(\begin{array}{ccc}
1 & 0 & 0 \\
0 & -1 & 0 \\
0 & 0 & -1
\end{array}\right) \\
& +m_{e e}\left(\begin{array}{ccc}
0 & \frac{\tan 2 \theta}{\sqrt{2}} & \frac{\tan 2 \theta}{\sqrt{2}} \\
\frac{\tan 2 \theta}{\sqrt{2}} & \frac{1}{2}-\frac{1}{2 \cos 2 \theta} & -\frac{1}{2}+\frac{1}{2 \cos 2 \theta} \\
\frac{\tan 2 \theta}{\sqrt{2}} & -\frac{1}{2}+\frac{1}{2 \cos 2 \theta} & \frac{1}{2}-\frac{1}{2 \cos 2 \theta}
\end{array}\right)
\end{aligned}
$$




$$
\begin{aligned}
& \pm m_{e e} \cos 2 \theta \frac{\varepsilon_{a t m}}{4}\left(\begin{array}{ccc}
0 & 0 & 0 \\
0 & -1 & +1 \\
0 & +1 & -1
\end{array}\right) \\
& +m_{e e} \frac{\varepsilon_{\text {sol }}}{8}\left(\begin{array}{ccc}
0 & \sqrt{2} \sin 2 \theta & \sqrt{2} \sin 2 \theta \\
\sqrt{2} \sin 2 \theta & -2 \cos 2 \theta & -2 \cos 2 \theta \\
\sqrt{2} \sin 2 \theta & -2 \cos 2 \theta & -2 \cos 2 \theta
\end{array}\right) \text {, } \\
& M_{B_{3}, B_{4}}=m_{e e}\left(\begin{array}{ccc}
1 & 0 & 0 \\
0 & 0 & -1 \\
0 & -1 & 0
\end{array}\right) \\
& +m_{e e}\left(\begin{array}{ccc}
0 & \frac{\tan 2 \theta}{\sqrt{2}} & \frac{\tan 2 \theta}{\sqrt{2}} \\
\frac{\tan 2 \theta}{\sqrt{2}} & -\frac{1}{2}+\frac{1}{2 \cos 2 \theta} & \frac{1}{2}-\frac{1}{2 \cos 2 \theta} \\
\frac{\tan 2 \theta}{\sqrt{2}} & \frac{1}{2}-\frac{1}{2 \cos 2 \theta} & -\frac{1}{2}+\frac{1}{2 \cos 2 \theta}
\end{array}\right) \\
& \pm m_{e e} \frac{\varepsilon_{a t m}}{4} \cos 2 \theta\left(\begin{array}{ccc}
0 & 0 & 0 \\
0 & 1 & -1 \\
0 & -1 & 1
\end{array}\right) \\
& +m_{e e} \frac{\varepsilon_{\text {sol }}}{8}\left(\begin{array}{ccc}
0 & \sqrt{2} \sin 2 \theta & \sqrt{2} \sin 2 \theta \\
\sqrt{2} \sin 2 \theta & -2 \cos 2 \theta & -2 \cos 2 \theta \\
\sqrt{2} \sin 2 \theta & -2 \cos 2 \theta & -2 \cos 2 \theta
\end{array}\right) \text {, }
\end{aligned}
$$

Here we recover the textures $\mathrm{C} 1$ and $\mathrm{C} 2$ of Altarelli-Feruglio [11](for the single maximal mixing case) and the absolute neutrino mass in the limit $\delta m_{\text {sol }}^{2}=\delta m_{\text {atm }}^{2}=0$ is $m_{e e} / \cos 2 \theta \sim m_{e e}$. The SMA solution could be obtained from this texture 0 but in this case, as already said above, the structure of the perturbation appears to be quite complicated, more complicated than from the texture in solutions $A_{1,2,3,4}$.

We then consider the solution around $\theta=\pi / 4$. In this case there are interesting zero textures. As explained above, the absolute neutrino mass, in the limit $\delta m_{\text {sol }}^{2}=$ $\delta m_{a t m}^{2}=0$, is now given by $\tilde{m} \equiv m_{e e} / \cos 2 \theta$. In this case $m_{e e}$ can be considered as a perturbation with respect to $m$ :

$$
\mathcal{M}_{B_{1}, B_{2}}=\tilde{m}\left(\begin{array}{ccc}
0 & \frac{1}{\sqrt{2}} & \frac{1}{\sqrt{2}} \\
\frac{1}{\sqrt{2}} & -\frac{1}{2} & \frac{1}{2} \\
\frac{1}{\sqrt{2}} & \frac{1}{2} & -\frac{1}{2}
\end{array}\right)
$$




$$
\begin{aligned}
+ & \tilde{m}\left(\begin{array}{ccc}
\cos 2 \theta & \frac{\sin 2 \theta-1}{\sqrt{2}} & \frac{\sin 2 \theta-1}{\sqrt{2}} \\
\frac{\sin 2 \theta-1}{\sqrt{2}} & -\frac{\cos 2 \theta}{2} & -\frac{\cos 2 \theta}{2} \\
\frac{\sin 2 \theta-1}{\sqrt{2}} & -\frac{\cos 2 \theta}{2} & -\frac{\cos 2 \theta}{2}
\end{array}\right) \\
& +\tilde{m} \cos ^{2} 2 \theta \frac{\varepsilon_{a t m}}{4}\left(\begin{array}{ccc}
0 & 0 & 0 \\
0 & -1 & +1 \\
0 & +1 & -1
\end{array}\right) \\
& +\tilde{m} \cos 2 \theta \frac{\varepsilon_{s o l}}{8}\left(\begin{array}{ccc}
0 & \sqrt{2} \sin 2 \theta & \sqrt{2} \sin 2 \theta \\
\sqrt{2} \sin 2 \theta & -2 \cos 2 \theta & -2 \cos 2 \theta \\
\sqrt{2} \sin 2 \theta & -2 \cos 2 \theta & -2 \cos 2 \theta
\end{array}\right), \\
\mathcal{M}_{B_{3}, B_{4}} & =\tilde{m}\left(\begin{array}{cccc}
0 & \frac{1}{\sqrt{2}} & \frac{1}{\sqrt{2}} \\
\frac{1}{\sqrt{2}} & \frac{1}{2} & -\frac{1}{2} & \\
\frac{1}{\sqrt{2}} & -\frac{1}{2} & \frac{1}{2} &
\end{array}\right) \\
& +\tilde{m}\left(\begin{array}{cccc}
\cos 2 \theta & \frac{\sin 2 \theta-1}{\sqrt{2}} & \frac{\sin 2 \theta-1}{\sqrt{2}} \\
\frac{\sin 2 \theta-1}{\sqrt{2}} & -\frac{\cos 2 \theta}{2} & -\frac{\cos 2 \theta}{2} \\
\frac{\sin 2 \theta-1}{\sqrt{2}} & -\frac{\cos 2 \theta}{2} & -\frac{\cos 2 \theta}{2}
\end{array}\right) \\
& \pm \tilde{m} \frac{\varepsilon_{a t m} \cos ^{2} 2 \theta}{4}\left(\begin{array}{lll}
0 & 0 & 0 \\
0 & 1 & -1 \\
0 & -1 & 1
\end{array}\right) \\
& +\tilde{m} \cos 2 \theta \frac{\varepsilon_{s o l}}{8}\left(\begin{array}{ccc}
0 & \sqrt{2} \sin 2 \theta & \sqrt{2} \sin 2 \theta \\
\sqrt{2} \sin 2 \theta & -2 \cos 2 \theta & -2 \cos 2 \theta \\
\sqrt{2} \sin 2 \theta & -2 \cos 2 \theta & -2 \cos 2 \theta
\end{array}\right),
\end{aligned}
$$

Here we recover the $\mathrm{C} 1$ and $\mathrm{C} 2$ textures of Altarelli-Feruglio (for the bimaximal mixing case) and see how the LMA solution can be obtained adding a perturbation from these textures which are the only ones where the neutrino masses in the limit $\delta m_{\text {atm }}^{2}=\delta m_{\text {sol }}^{2}=0$ doesn't coincide with $m_{e e}$ but can be much larger than $m_{e e}$. Therefore these textures are the most relevant ones for dark matter. In the limit of exact bimaximal mixing and $m_{e e}$ fixed this neutrino mass is infinite. However as Eqs. (29) and (30) show, from these two textures we can get the LMA solution (with $\theta<\pi / 4$ ) from the exact bimaximal case considering $m_{e e}$ as a perturbation with respect to $\tilde{m}=m_{e e} / \cos 2 \theta$. Because of this involved nature of the solution, we could get a LMA solution in this way even if the 11 element vanishes in the zero texture, which wouldn't be so easy to see starting directly from the texture zero as in the analysis of Ref. [11]. 
- The inverse hierarchy case: We could have an inverse hierarchy solution, only if $m_{e e}$ turns out to be not much larger than the lower edge of the range of Eq. (1)), i.e. $m_{e e}=0.05 \mathrm{eV}$. This is possible only with solutions $B_{2,4}$, because as with solutions $A_{2,4}, \delta m_{a t m}^{2}$ has to come with an opposite sign with respect to the term in $m_{e e}^{2}$ in $m_{3}$, Eqs. (17)-(18). For these solutions $B_{2,4}$ an inverse hierarchy requires $m_{e e}^{2} \sim$ $\cos ^{2} 2 \theta \delta m_{\text {atm }}^{2}$. For the central values $\delta m_{\text {atm }}^{2}=2.9 \cdot 10^{-3} \mathrm{eV}^{2}$ [5] and $\tan ^{2} \theta=0.37$ [6], this requires for the LMA solution $m_{e e} \sim 0.025 \mathrm{eV}$. Varying $\tan ^{2} \theta$ within the range $0.2-0.8$ [6] and $\delta m_{\text {atm }}^{2}$ within the range $(1.5-4.8) \cdot 10^{-3} \mathrm{eV}^{2}$ this requires:

$$
5 \cdot 10^{-3} \mathrm{eV}<m_{e e}<5 \cdot 10^{-2} \mathrm{eV} \text { (for solutions } B_{2,4} \text { and LMA) }
$$

For the SMA solution since $\cos 2 \theta$ is very close from unity the corresponding allowed range coincides with the one of Eq. (23). Note finally that if we develop the mass matrix around $\theta=0$ and $\theta=\pi / 4$, considering $m_{3}$ and $\sqrt{\delta m_{\text {sol }}^{2}}$ as perturbations with respect to $\tilde{m}$ and $\sqrt{\delta m_{\text {atm }}^{2}}$ we obtain the following zero textures:

$$
\left(\begin{array}{ccc}
1 & 0 & 0 \\
0 & -1 / 2 & -1 / 2 \\
0 & -1 / 2 & -1 / 2
\end{array}\right), \text { and }\left(\begin{array}{ccc}
0 & 1 / \sqrt{2} & 1 / \sqrt{2} \\
1 / \sqrt{2} & 0 & 0 \\
1 / \sqrt{2} & 0 & 0
\end{array}\right) \text {, }
$$

respectively (that is to say the textures B1 of Ref. [11]).

7) Ambiguities of the perturbations: In Eqs. (19)-(22) we have given the structure of the perturbations which has been determined directly by Eqs. (7)-(18). They are the minimal perturbations which have to be added to give the atmospheric mass difference together with the solar angle and solar mass difference. It must be noted, however, that these perturbations are not unique. For example in Eq. (19) we could perfectly add a perturbation which is simply proportional to $\varepsilon_{a t m}$ and the identity matrix. To add this perturbation corresponds to a redefinition of the mass scale of the zero texture by a small amount. More generally there exist perturbations proportional to $\varepsilon_{\text {sol }}$ we could add which are not proportional to the zero texture and which could leave the condition $m_{2}^{2}-m_{1}^{2}=$ $\delta m_{\text {sol }}^{2}$ unchanged while changing the scale of the zero texture or the atmospheric mass difference by a negligible term proportional to $\delta m_{\text {sol }}^{2}$. To add such a perturbation leads to a mass matrix still fulfilling all the experimental constraints, but with another structure of perturbation. Note that the atmospheric mass difference is anyway far from being determined at the level of accuracy of the solar mass difference. This leads to some freedom in the perturbations which exists also for perturbations proportional to $\delta m_{a t m}^{2}$. We leave this discussion to a subsequent publication. Here we gave just the "minimal" perturbations 
and we do not consider these extra perturbations which are not necessary to satisfy the experimental constraints.

Although the atmospheric neutrino mixing is allowed to be in the range of $\sin ^{2} 2 \theta_{\text {atm }}=$ $0.87-1$, we considered only the maximal mixing and not the entire range. This and the possible small deviations from the constraint $U_{e 3}=0$ may allow for few more perturbations, which we disregarded here.

8) Summary: In summary, instead of starting the analysis from the possible textures, we started with the experimental inputs assuming in addition that $m_{e e}$ is larger than the neutrino mass differences. We then determined the neutrino mass matrices and in the process got the possible textures. We found that, for maximal atmospheric mixing and $U_{e 3}=0$ and for a given set of values of $m_{e e}, \delta m_{\text {sol }}^{2}, \delta m_{a t m}^{2}$ and $\theta$, there are 8 solutions for the mass matrix. Among them the solutions $A_{1}$ to $A_{4}$ have the interesting property to allow a decomposition which is just a zero texture depending only on a single mass scale, plus a perturbation depending only on $\delta m_{\text {atm }}^{2}$, plus a perturbation depending only on $\delta m_{\text {sol }}^{2}$ and $\theta$. There is also a set of solution $B_{1}$ to $B_{4}$ whose structure of perturbations is more complicated but which allow neutrino masses larger than $m_{e e}$. All solutions and especially $B_{1,2,3,4}$ lead naturally to neutrino masses relevant for dark matter. For solutions $B_{1,2,3,4}$, a lower $m_{e e}$ and a higher $\cos 2 \theta$ is preferred by the dark matter fits. We observed also that for solutions $B_{1,2,3,4}$ there is a continuum of possible zero textures. For each solution we displayed the allowed ranges for the mass eigenvalues together with the corresponding zero texture and we showed how, in the degenerate neutrino mass scenario, the perturbations come up to give the full mass matrix.

\section{Acknowledgements}

We thank H.V. Klapdor-Kleingrothaus and U. Sarkar for several useful discussions and their encouragements. We also acknowledge useful comments from M. Raidal and J. Orloff. This work was supported by the TMR, EC-contract No. ERBFMRX-CT980169 (EuroDa $\phi$ ne).

Last minute note: in the process of submitting this manuscript, some of the results derived here turn out to have been presented independently in Refs. 22, 23. 


\section{References}

[1] Super-Kamiokande Collaboration : Y. Fukuda et al, Phys. Rev. Lett. 81, 1562 (1998); Phys. Lett. B433, 9 (1998); Phys. Rev. Lett. 85, 3999 (2000).

[2] Super-Kamiokande Collaboration: Y. Fukuda et al, Phys. Rev. Lett. 81, 1158 (1998); Phys. Rev. Lett. 86, 5656 (2001); SNO Collaboration: Q.R. Ahmad, et. al., Phys. Rev. Lett. 87, 071301 (2001).

[3] K2K Collaboration: S.H. Ahn, et. al., Phys. Lett. B 511, 178 (2001); K. Nishikawa, im Proc. of the Int. Europhysics Conf. on High Energy Physics (Budapest, Hungary, July 2001); C.K. Jung, in Proc. of the XX Int. Symp. on lepton and Photon Interactions at High Energies (Rome, Italy, July 2001).

[4] LSND Collaboration: C. Athanassopoulos, et. al., Phys. Rev. Lett. 77, 3082 (1996); Phys. Rev. Lett. 81, 1774 (1998).

[5] G.I. Fogli, E. Lisi and A. Marrone, hep-ph/0110089.

[6] J.N. Bahcall, M.C. Gonzalez-Garcia and C. Peña-Garay, hep-ph/0111150.

[7] CHOOZ Collaboration: M. Appollonio et al, Phys. Lett. B 420 (1998) 397; Phys. Lett. B 466 (1999) 415.

[8] H.V. Klapdor-Kleingrothaus, A. Dietz, H.L. Harney and I.V. Krivosheina, Mod. Phys. Lett. A16 (2001) 2409.

[9] C. Augier for the NEMO collaboration, CIPANP 2000, AIP Conf. Proceed., vol 549 (2001) 819; A. Alessandrello et al. (CUORE et Cuoricino collaborations), hepex/0201038; H. Eijiri et al. (MOON collaboration), nucl-ex/9911008; H.V. KlapdorKleingrothaus et al. (GENIUS collaboration), MPI-H-V26-1999, published in Tegernsee 1999, Beyond the desert 915-1014, hep-ph/9910205.

[10] H.V. Klapdor-Kleingrothaus, H. Paes and A.Yu. Smirnov, Phys. Rev. D 63 (2001) 073005; H.V. Klapdor-Kleingrothaus and U. Sarkar, Mod. Phys Lett. A16 (2001) 2469.

[11] G. Altarelli and F. Feruglio, Phys. Rep. 320 (1999) 295.

[12] N. Cabibbo, Phys. Lett. B72 (1978) 333; A. De Rujula, M.B. Gavela and P. Hernandez, Nucl. Phys. B547 (1999) 21. 
[13] R. Barbieri, G.G. Ross and A. Strumia, JHEP 20 (1999) 9910.

[14] F. Vissani, hep-ph/9708483; R. Adhikari and G. Rajasekaran, Phys. Rev. D 61 (1999) 031301(R); H. Georgi and S.L. Glashow, Phys. Rev. D 61 (2000) 097301.

[15] V. Barger and K. Whisnant, Phys. Lett. B456 (1999) 194.

[16] R.A.C. Croft, W. Hu and R. Dave, Phys. Rev. Lett. 83 (1999) 1092.

[17] D.J. Eisenstein, W. Hu and M. Tegmark, astro-ph/9807130.

[18] W. Hu, D.J. Eisenstein and M. Tegmark, Phys. Rev. Lett. 80 (1998) 5255.

[19] V. Lobashev et. al. Phys. Lett. B 460 (1999) 227; Ch. Weinheimer et. al. Phys. Lett. B 460 (1999) 219.

[20] E. Gawiser and J. Silk, Science 280 (1998) 1405; J. Primack, astro-ph/9707285.

[21] R.E. Lopez, astro-ph/9909414.

[22] V. Barger, S.L. Glashow, D. Marfatia and K. Whisnant, hep-ph/0201262.

[23] F. Feruglio, A. Strumia and F. Vissani, hep-ph/0201291. 